\title{
Fabricating Performance: Reciprocal Constructs of Dance Notation
}

\author{
Syuko Kato Westby ${ }^{1}$ - Ruairi Glynn ${ }^{1}$
}

Published online: 10 November 2017

(C) Kim Williams Books, Turin 2017

\begin{abstract}
The analogue and digital notational systems for documenting choreographic movement provide alternative strategies for spatial design. These strategies overlap architectural design and dance choreography to explore reciprocal exchanges regarding the body, geometry and methods of spatial notation. Analogue and digital notational systems are compared to illustrate a change where the notation is fed back to the performer and used as inspiration for further iterative performances. Whilst the use of analogue notation systems supports the criterion of fundamental design, they have limitations which are overcome with the use of flexible digital systems that more readily adapt to change and interrelate to a dancer's intentions for movement creation. Performance-driven fabrication explores the practical application of this process. Two stages of 'Performance-driven design' and 'Data-driven fabrication' are combined, resulting in a spatial design and construction system that incorporates interactivity between human and robotic performers.
\end{abstract}

Keywords Notation · Choreography · Performance $\cdot$ Fabrication · Robotics

\section{Introduction}

The relationship between architecture and choreography in the construction of space has been a constant source of discussion between architects and choreographers since the 1900s. Reciprocal exchanges of language to discuss the body and its geometry have been assimilated, often altering the meanings of these terms and offering designers and artists new insights into the creative process.

Syuko Kato Westby

katosyuko@gmail.com

1 Bartlett School of Architecture, University College London, 22 Gordon Street, London WC1H 0QB, UK 
However, architectural drawings rarely capture moving factors; typically illustrating frozen conditions, they fail to convey changing environments of human life and its energy.

The importance of transitions in space, according to the time changes, was stressed as early as 1988. Siegfried (1988) questioned the value of art, claiming that only tangible objects had been focused on and that movement or changes across time in space were not highlighted. There have been practitioners who responded to these intangibles in their art, which can be seen in the process of its making. Vladimir Tatlin introduced the idea of framing time with his models 'Counter-relief (1911), 'Hanging Construction (1920)', and 'Monument to the Third International (1919-1920)'. Theoretical architectural research on design approaches in the 1960s and 1970s included Cedric Price's 'Fun Palace' and Archigram's 'Plug-in and Walking Cities'. This discourse presented a major challenge to traditional paradigms of movement notation in architecture. These examples distinguish their physical models and drawings from conventional methodologies, evaluating the process of translating movement information.

Digital notation has the potential to translate various dynamics of movement into architecture. In the context of generative design, digital notation could play an important role in incorporating design principles, in which the 'quality' of changes may be reflected, to address the question of notating moving elements in the area of dance choreography and architectural design. This could be called a methodology creation for qualifying movement in space.

Movement, although it is intangible, plays an important role as a description of space. To analyse this in both the dance choreography and architectural fields, the interpretation of movement and its quality in each context is essential in order to capture these factors and re-structure them in detail. Such quantification and qualification is indispensable when notating movement and discussing its form.

By cross-referencing drawings and notations developed by practitioners who significantly contributed to the development of both fields in architecture and dance choreography, this paper aims to seek alternative design tools that employ a concept of movement in relation to its transformational process. Subsequently the method is applied to the problem of integrating the creation of spatial environments. It considers the role of notation and suggests that building a common language as a design vocabulary, drawn from the fields of dance and architecture, could enhance the quality of experience for users. Through the inhabitants' involvement in space, their senses can be challenged by their choice of journey and how they relate to their physical surroundings. More specifically, the methodologies for translating this 'creative' approach to design space are analysed from a viewpoint of Euclidean geometric exactness, rhythmic pattern and perceived 'quality' of movement.

The aim is to provide a new paradigm for designers, with a novel approach to quantifying and qualifying the adaptability and responsiveness of a space. This is done in relation to real-time experience, and the changing condition of space. Methodologies for capturing segments of movement, defined by quality of movement, are discussed relative to the potential for them to re-inform design processes. In this context, 'performance' refers to the happenings and the events that 
can be considered to activate a space, whether it is in the process of making a piece of dance choreography, or the process of cultivating ideas for architectural design.

The paper is structured in three main sections. First, movement is defined in order to clarify the intentions of notating this invisible property. The limitation of analogue notation methodologies is reviewed. Second, digital notation systems are introduced as potential alternative design tools. The objectives of prospective design research through the use of digital data transcription in choreography are introduced. Third, a design investigation conducted in parallel to this research paper is presented to demonstrate a scenario in which the digital movement scores initiate the process of design and architectural fabrication.

\section{Terminology}

\section{Notation}

Notation is a measurable factor in the process of design. It provides a medium for the exchange of information, and facilitates the negotiation of shared goals among those who may be involved in the creation of space. In dance, notation systems were invented in order to record movement for later use, and subsequently have become part of the creative process. An overview of terminologies used in notation systems, such as diagrams, symbols, mapping and scripting, are classified in Fig. 1.

Digital notation employs numerical information. It embraces input and output of data, according to the conditional change in space. It designates the time scale therefore it can be flexible in notating movement information. With digital notation systems, the potentials of generating space according to movement information can be explored.

Choreographic notation may specifically suggest creative approaches in dance choreography and spatial design. It allows performers to add annotative explanation, and the original notation develops each time it is performed. Through repetition, notation is denoted and movement is further suggested to the latter dancers in a
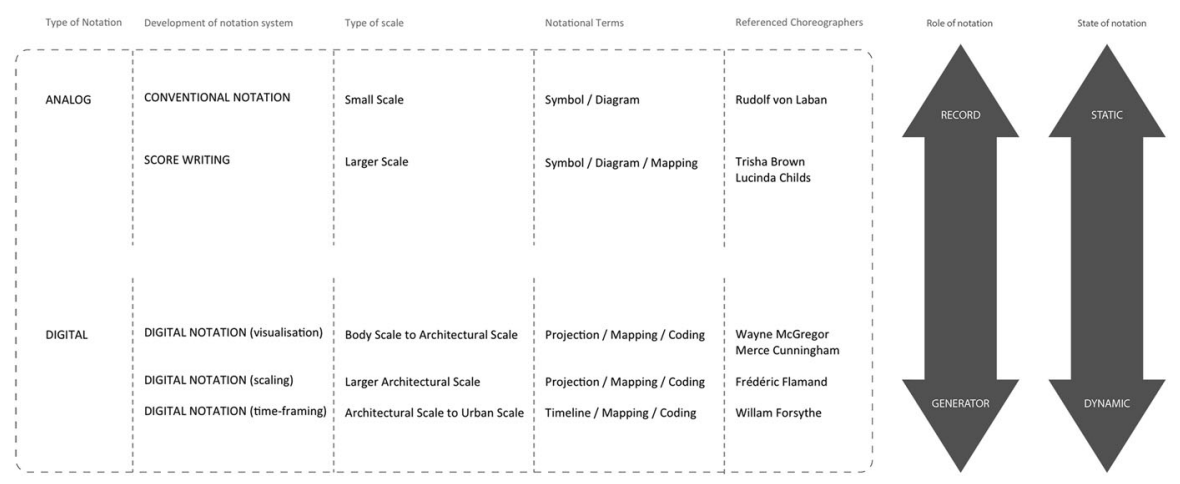

Fig. 1 Notation classification 
same but different form. Notation gradually grows, reflecting dancers' intents and engagement to space.

\section{Performance}

Iterative performance is the quality of executions, acted in a repeatable manner. It provides rules that suggest choreographic structures to be formed alongside the procedures. When repetition is provided each time, fresh information is added on to the original statement. Further initiative information is provided to encourage proceeding to the next sequence.

Within a design process, transformational procedures enable mixed elements to be exchanged and demonstrate progressions that occur in sequential order. They are also replicable under certain conditions of commands in iterative rules. Ultimately, outputs deliver sources to be used as inputs, generating the design process in a loop format.

Inhabitable space can be defined as a three-dimensional enclosure, to which one may relate using body movement and interaction (Bacon 1974; cited in Salingaros 1999). It is formed with the geometry and spatial information of urban space. Decisions informing path structures rely on the observers' interactions (Salingaros 1999). In this paper, the relationship of the occupier to inhabitable space is studied through iterative performances.

\section{Qualities of Movement}

Qualities of movement in dance can be classified into two categories: phenomenological and ethological (Siegfried 1988). The former is usually perceived by observing the human body in motion. It has the quality of being persistent in situational happenings, and generates movement with rhythm, patterns and in connection to the body and geometry. The latter, on the other hand, is the analysis of movement itself. Ethology can be included in an analysis of design procedures as it contributes to the ritualisation or evolution of movement. However, this paper focuses on the approach of phenomenology in its relationship to design in architecture.

Principles of mapping 'quality' in movement are frequently explained with a series of Étienne-Jules Marey’s work. Such early attempts, which envisage the invisible dynamics around the body, initiated the further development of creative works where dance choreography and architecture overlap.

Rudolf von Laban (1879-1958), who studied dance and architecture, introduced the 'quality' of movement in relation to spatial interaction in order to develop an understanding of the interconnection between geometry and movement, and to accommodate physical motion to a notation system. In the context of movement in structures, Laban conducted research on an icosahedron model (Fig. 2), and through this model, he produced written diagrams, entitled Labanotation, which have given many choreographers and dancers the opportunity to build their own notation systems based on Laban's studies. 

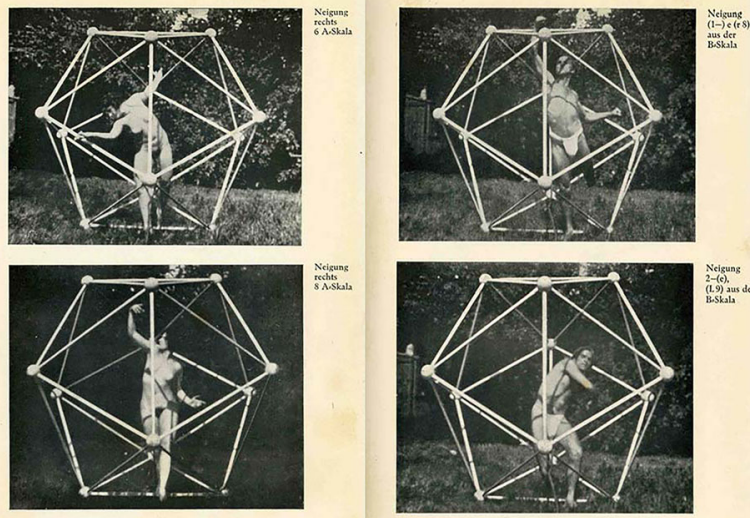

Fig. 2 Image from R. Laban, 1926, Choreography. Jena: Eugen Diederichs

What is observed as quality of movement from Laban's choreographic research is Euclidean geometric exactness in respect to body structure. Movement is integrated through translation tools, such as body-sized physical models and a notation system applied to paper. The perceived quality is translated into rhythmic patterns, providing information that can be referred to for future use.

In the same period of time, Euclidean geometry was also explained as fundamental to quality of movement in connection with the structural formation known as 'Jitterbug'. Buckminster Fuller (1895-1983) explained this with his structural model, within which the mutual transformations between the icosahedron, cuboctahedron, tetrahedron and octahedron are accommodated (Fig. 3). Schwabe described Fuller's structure as "a fluid motion in which one body dissolves into another, one spatial figure develops from the other, just like in a dance" (Schwabe 2010).

In the search for alternative tools for defining choreographing movement, it is essential to intentionally identify the information of movement (i.e. speed, accent), as well as the physical form of it (i.e. geometry, position, angle).

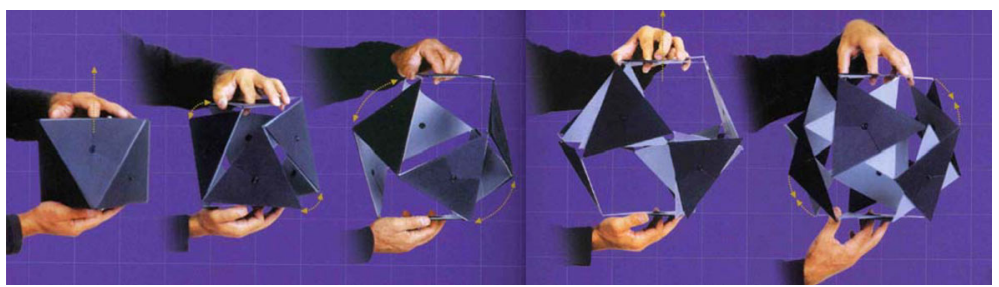

Fig. 3 The Joe Clinton Jitterbug Transformation (in C. Schwabe, 2006, Geometric Art. Tokyo: Kousakusha. pp.194-195). Reproduced by permission 


\section{The Notation of Choreography and Architecture?}

In the search for defining alternative tools for choreographing movement, it is essential to identify the information of movement (i.e. speed, accent), as well as the physical form of it (i.e. geometry, position, angle). Architects and choreographers' attempts at analogue notation are analysed.

\section{The Role of Notation in Architecture}

Drawings and descriptions of designs give architects the means to mediate the relationship between themselves, client and building, setting the rules for negotiation between concept and realisation. Gage argues for architects to consider behavioural rules in design and to consider how adaptability and habitation affect occupation of designed space. Notation of movement here may help to predict behaviour (Gage 2007).

Notation methods are pivotal in some information diagrams. In the 'Fun Palace' Cedric Price (1934-2003) used mechanical systems to programmatically actuate his building. He used diagrams to explain the movement and behaviours of people and services and notate habitable conditions in the context of a moving building with free-flowing people (Fig. 4). Notation allows coherent communication of function and experience.

Following Price's approach, Tschumi (1996) devised modes of notation derived from choreography to expose the complexity of phenomena in architecture and culture that he wished to communicate.

Here, movement becomes contextual information capturing the implications of spatial arrangement on participation and occupation. The essence of movement

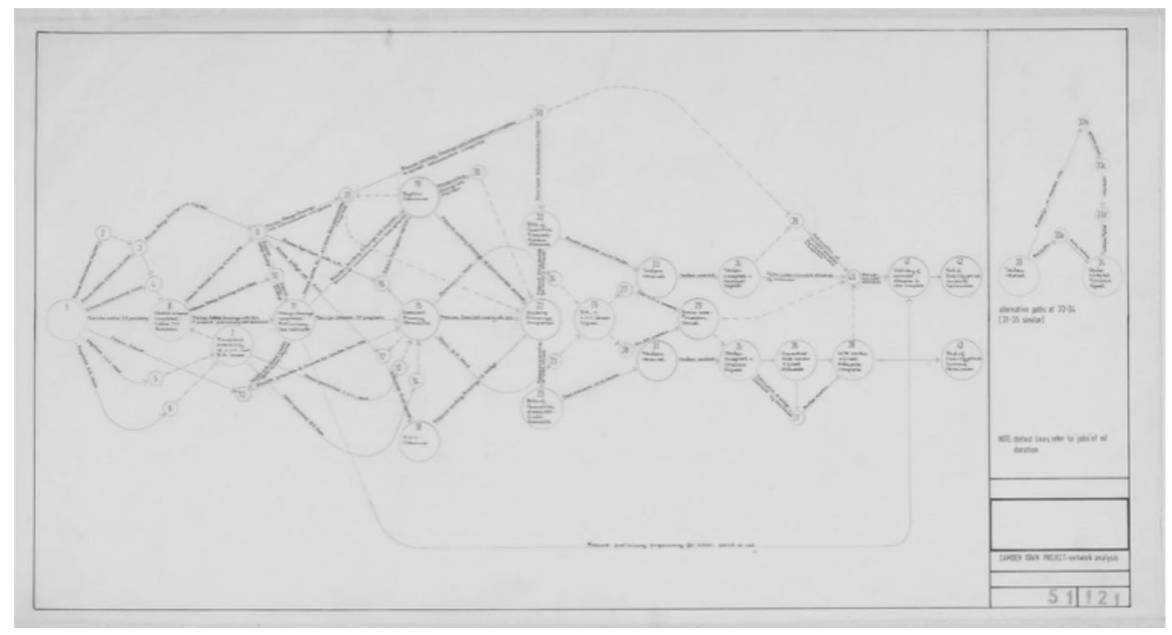

Fig. 4 Fun Palace: network analysis (by Cedric Price, 1964-1965. Cedric Price fonds, Canadian Centre for Architecture. Available from: http://www.cca.qc.ca/en/route/rk/collection-object-309847). Reproduced by permission 
appears in drawings and diagrams, but the dynamics of interaction between user and space fail to be captured.

\section{The Role of Notation in Dance Choreography}

Transcribing dance challenges the creative process of choreography. An analogue process traces a transient segment into a frozen state on paper. Languages have developed to expediently notate movement. Trisha Brown, in Locus, co-opted mathematics to describe movement. Her scores required dancers to move sequentially through numbered points in a cubed format space.

In contrast Lucinda Childs' notations simultaneously arrange multiple dancers' movements in a larger space. As Weinstein explains, this successfully organises rhythms and patterns to be executed, as opposed to Labanotation, which "does not convey full scalar spectrum of information" (Weinstein 2013). This highlights the discrepancy in notations and differences in transcribing movements.

Such attempts show notations cannot simply extract geometrical position information and relay it as two-dimensional records. Where Brown annotates quantitative factors in space, Childs' choreographic procedure presents contextual movement in patterns to be interpreted. Analogue techniques now form the basis for notation but have limitations in specificity of movement and scope with multiple dancers.

\section{Measuring the Quality of Movement}

\section{Digital Notation}

Negroponte (1970) was keen to use new digital notation methods to help visualise movement and activity in the city. His diagram uses visual variables as a means of handling large amounts of information. It was illustrated on a two-dimensional plane; however, informative features of a multitude of forces were depicted in order to exploit the computer's capability of capturing transitions of information. The introduction of script writing, using variables as a source for handling a large amount of information, integrating the possibilities of movement translation in changing an environment to a visual or physical one.

\section{Visualisation}

The use of a digital interface to translate creative sources for dance choreography is observed through visual tool creation. In Wayne McGregor's recent piece 'Atomos' (2013), choreographic language as digital script writing was explored. Using numerical data transfers, visual artists Marc Downie (OpenEndedGroup) and Nick Rothwell (CASSEIEL) created the tool 'Becoming' (Fig. 5a, b). Performers use the feedbacks of computer-based visuals, which reflect the changes of colours in the referenced film, as inspiration for further movement. Using these creative tools, the concept of biometric data is transferred through a dancer's body movement by capturing biological rhythms around their body. 


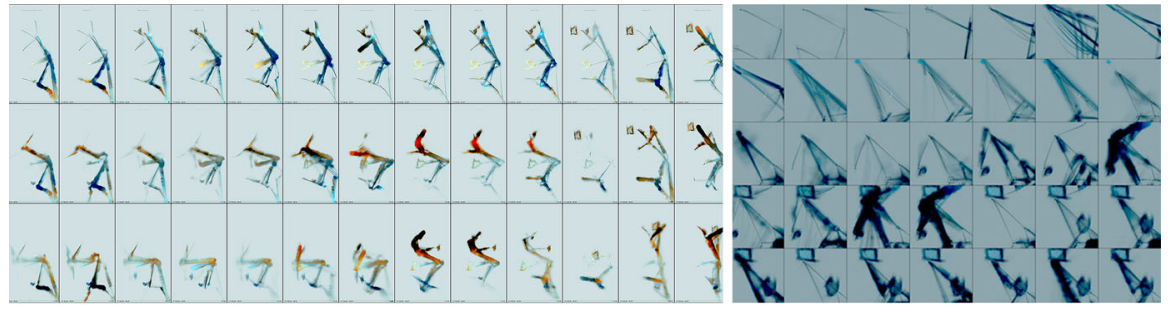

(a)

(b)

Fig. 5 a OpenEndedGroup. 2011. Image for Becoming. b OpenEndedGroup. 2011. Image for Becoming. Available from: http://openendedgroup.com, Reproduced by permission

Such an example of work that is attempted through the collaboration between digital artists as well as choreographers and architects has integrated the role of scores with procedures for designing performance and spatial choreography.

\section{Scaling}

The above project enabled designers and choreographers to use an external variable to conduct a dance, rather than didactic notation. It frees the system from scale.

In Moving Target (1996), by Diller + Scofidio and Frédéric Flamand, the challenge of 'scaling' and 'de-scaling' can be seen. Dancers and projections simultaneously occupy the arena, also combining a reflected stage and projected dancers. Encounters between the real dancer's movement and the virtually reflected bodies are merged, while dancer's movements are governed by moving projection targets. Several technologies support an optimised scenography creating deception and dynamic responses.

Here technology turns space into an instrument to be played in a dynamic actthe quality of body movement is reshaped each time. Ultimately, they become observable objects as well as being independent from the original scale. Collard explains "By refusing stable reference points, it shifts our attention from artistic product to signifying process" (Collard 2015). The evidence of this piece shows how precision is perceived in different individuals.

\section{Time-Framing}

A timeline offers a way to recognise periodicity within a score. The digital notation allows information on the score to recur freely, but allocates it within a dynamic framework using a set of rules. For Forsythe's piece One Flat Thing (2013), 'synchronous objects' (Fig. 6a, b) were developed with digital artists at Ohio State University. Codes give hierarchy and individual tasks. It frees dancers to produce individual movement whilst attention can still be paid to the collective movement. Groves describes how these scores "do not transcribe movement, but call attention to how ideas produce movement and how movement occasions ideas" (Groves et al. 2007). 


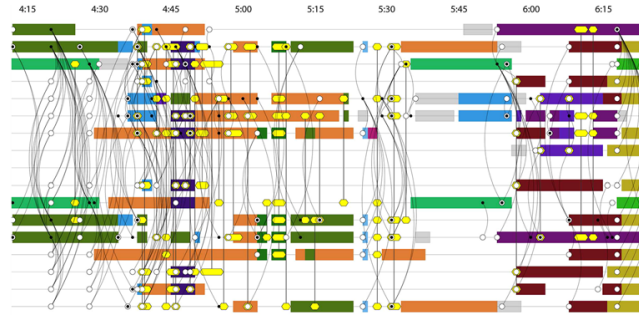

(a)

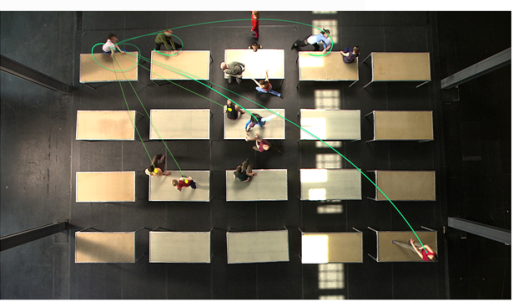

(b)

Fig. 6 a Synchronous Objects Project, The Ohio State University and The Forsythe Company, 2013, Cues and Themes, b Synchronous Objects Project, The Ohio State University and The Forsythe Company, 2013, Cueing System. Reproduced by permission

The timeline can be used in architecture as a tool for organising and situating conditions of changing environments. Kamvasinou (2010) demonstrates this in her 'Stansted project' (Fig. 7). Notation focuses on the experience of space, rather than object. Information about a site is more precisely identified as transitional factors occurring in time. Time-based interfaces offer routes for individuals. Digital processes help manage this plethora of data in future design processes.

Sparacino stresses that architectural thinking and practice have been shaped and driven in accordance with the development of technology (Sparacino 2002). In her 2002 paper 'Narrative Space', she points out how technological platforms affect inhabitants' experience of surrounding space, such as the cultural space, the local, the city and the private house. The importance of developing tools for architects is emphasised through the research on her media-augmented performance at the MIT Media Lab since 1994.
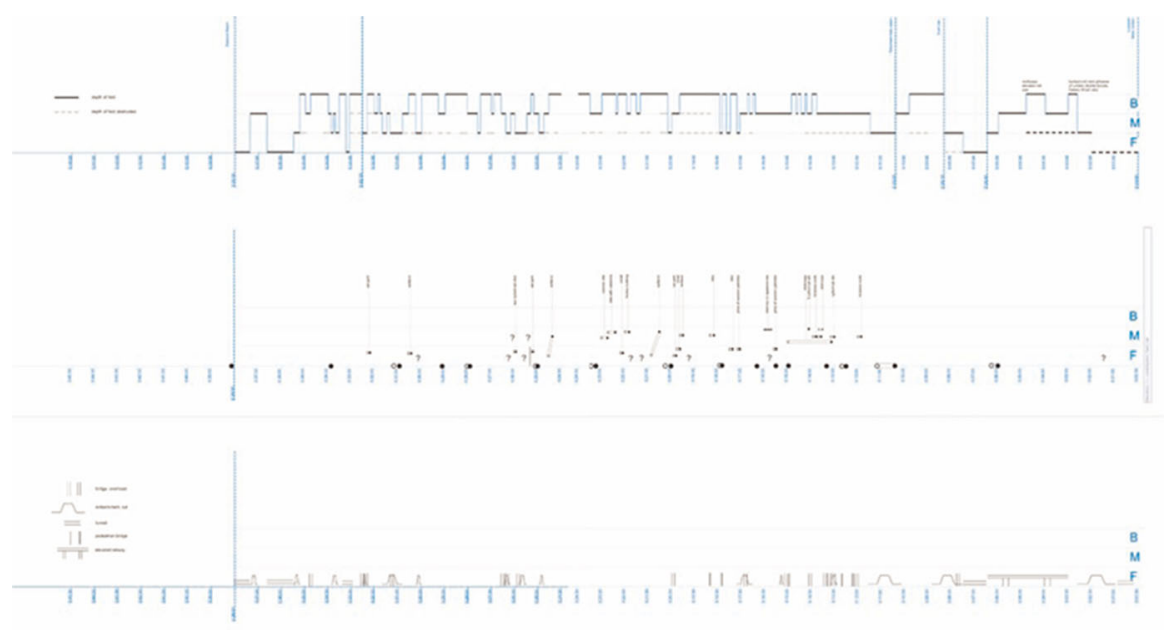

Fig. 7 Kamvasinou (2010), notation timelines and the aesthetics of disappearance (in: The Journal of Architecture, 15(4), 397-423). Reproduced by permission 
Given the exactitude of the computer-orientated inventions, one can see that it is possible to notate performance in architecture without losing information in the translation process. In other words, architects and designers can design precisely structured forms, incorporating a transformational 'quality' of movement.

The studies evaluating perceived 'quality' of movement have brought unique discoveries to designers' and choreographers' creative idea exchanges. These approaches of transferring analogue movement information to digital design resources offered the users' and performers' participatory activities, generating their creative mind in both choreographic process and design of these spaces.

\section{Fabrication of Movements}

When movement information appears on the process of formation and deformation, this interactive environment can be defined as Performance of Architecture. In contrast to movements that are statically described in space, the previous examples of digitally understood transitional data could also directly structure the geometry and suggest reality and liveliness to its inhabitants.

Goulthorpe notes how fabrication processes offer designers the opportunity to gain new insights for their design process. At his practice dECOi, Ether/I (1995, Fig. 8) was a built movement form derived from dance. As a tribute to choreographer William Forsythe, their analysis of the relationship between movements and its intention were successfully transcribed into an aluminium structure. Forsythe's duet piece was videorecorded multiple times before digital models on computer were developed. The repetitions of a dance are interpreted as qualities such as uncertainties, hesitations and tempo changes (Bonnemaison and Eisenbach 2009). Goulthorpe explains this as 'the necessary difference or error or performance'.

While 'Ether/I' explored a production of invisible factors, dECOi would later develop a more 'mutable' process through their 'Aegis Hyposurface' project in collaboration with Deakin University. Developed through the experience of Ether/I, it focuses onto constructing conditional changes, instead of capturing momental happenings which are appeared to be rigid objects. "The characteristics of generative systems, in which parameters instead of stable relationships were the currency" (Goulthorpe et al. 2001). Using an array of linear actuators, a dynamic
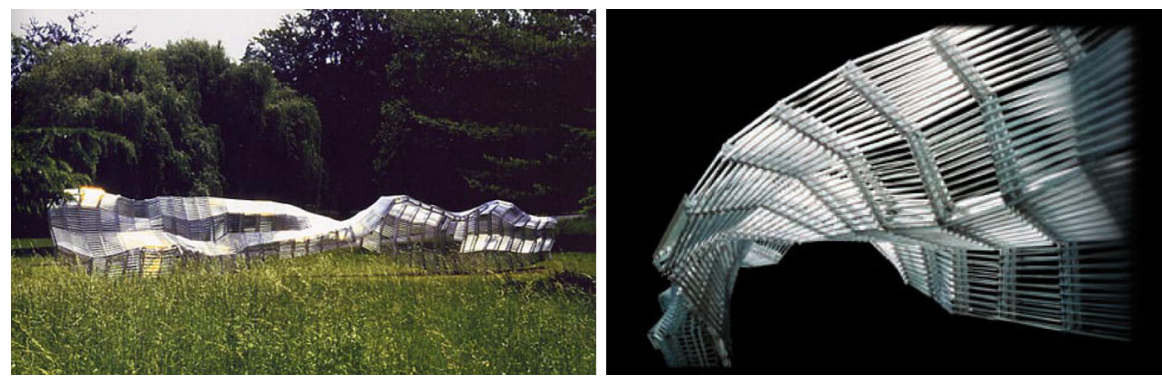

Fig. 8 M. Goulthorpe, dECOi, 1995. Ether/I. Reproduced by permission 


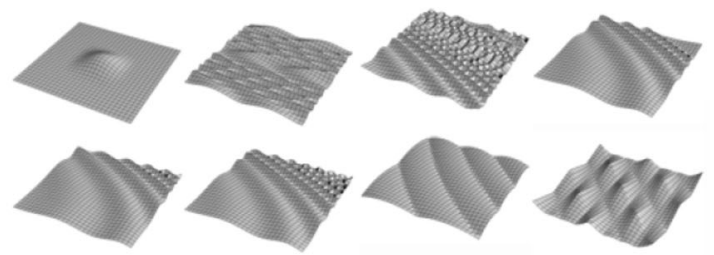

Surface formulae

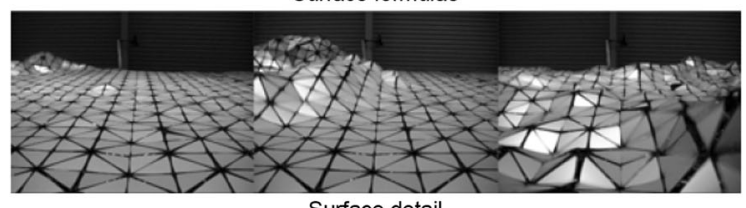

Surface detail

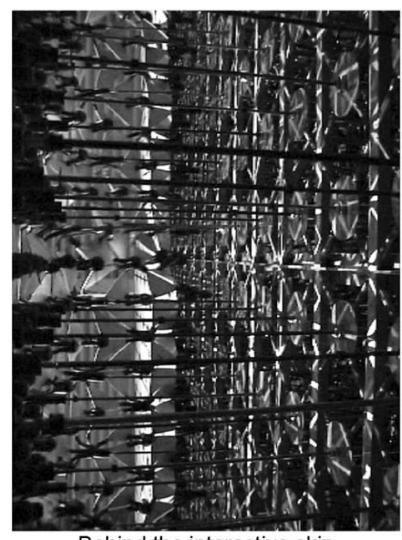

Behind the interactive skin

Fig. 9 HypoSurface: A Dynamically Interactive Architectural Surface, a New Medium for the Plastic Arts (images from Goulthorpe et al. 2001, 346), Reproduced by permission

surface that reconfigures in response to the environmental change was developed. It raised interesting questions about what forms of design methods and creative processes were appropriate when engaging with live data (Fig. 9).

The process of translating digital information to physical form offered considerable creative opportunities for designers as an open-ended tool for collaboration. However, the physical results of these transformations were ephemeral, leaving no architectural artefact documenting sequences of motion.

\section{Fabricating Performance}

In our research project, Fabricating Performance, developed at the Interactive Architecture Lab, Bartlett, UCL, digital scores are in dialogue with a fabrication system (Fig. 10). An iterative feedback loop bridges analogue and digital processes, dance, motion capture, digital notation and robotic construction.

The primary aim of the work is to explore the potential of digitalised notation for implementing fabrication of analogic space from imagery of dance movement. Both the rendering of the intentions of performers and the 'creativity' at the point of translation of each process will be examined. This work focuses on capturing what has been discussed as the 'quality of movement' through the digitally written scripts, and tries to translate it to geometrical form.

The design proposal combines two stages of performance-driven design and datadriven fabrication, processing itself in order to realise physical results through information from the performance. In this scenario, design is conducted using data extracted from a procedure of performance, representing the movement quality, and a physical inhabitable space is constructed from instant feedbacks that are derived from the dancer's movement. During this procedure, 'iterative' translation between the analogue and the digital processes takes place in the following three forms: 

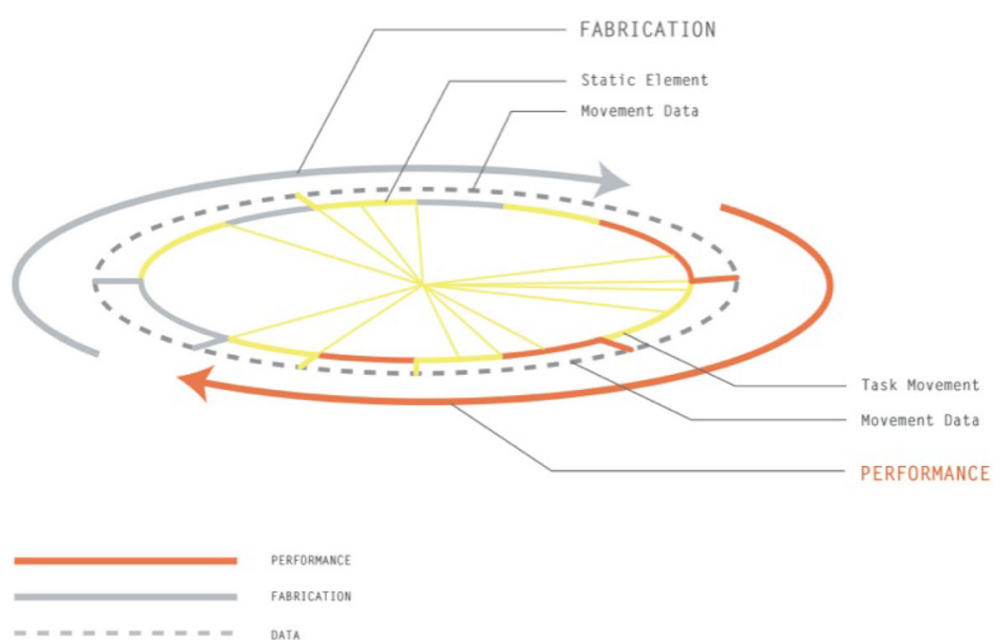

Fig. 10 Performance-driven fabrication scenario

analogue dance (body movement in space); digital notation of the dance; analogue spatial construction.

\section{Performance-Driven Design}

\section{Analogue Dance}

Looking at the utilisation of digital systems for input and output of information, the initial study focused on obtaining aspects of improvised dance movement. Rather than attempting to capture a continuous flow of information on the sequence, the instance at the beginning and the end of each movement sequence are extrapolated as movement data. These extrapolations of movement were considered to hold the condensed information regarding the dancer's intention in producing each sequence. At an introductory point of each sequence, the implication of notational vocabulary is used to start a sequential movement. Similarly, in this way, at the end of the movement, an array is intended to end with an instructive movement for the second dancer to start a sequence. Some study models were produced from these fragments of motion (see Fig. 11) and analysis is made of how these extrapolations of analogue information could be translated to digital ones.

As a result, some possibilities for capturing intentional movement were observed (i.e. weight and shift, flow and speed or length in geometry). The dancer's engagement with space around the body is represented by the figures as 'positive space', which imply the perception of space through the improvisation.

\section{Digital Notation}

The next challenge was to design rules in order to translate these intentional movements to digital formats. Based on the observed "perception of space" through 

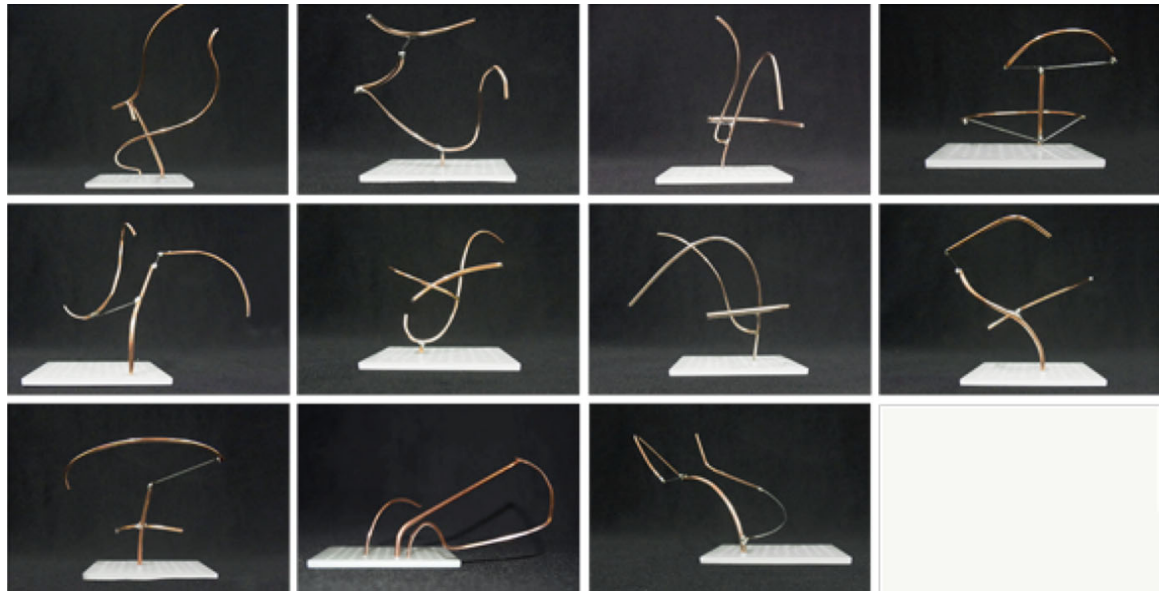

Fig. 11 Dancer's engagement to space is represented by the figures as 'positive space'

the analogue dance investigation, further choreographic studies were conducted in order to measure the 'quality' of movement more precisely. A movement tracking system, 'Optitrack' (NaturalPoint, Inc.), is employed to capture information on bodily motion, and the collected data is analysed with Python code scripting to develop a dialogue in-between dance, choreography and architecture. At each stage of the procedure, studies are focused on the hypothesis that a design system can be implemented by generative procedures in which the notated scores are processed by computational technology (Fig. 12).

Following is the analysis of data collected from 'Improvised movement', 'Rule based movement' and 'Choreographed movement'. Recorded movement is visualised as geometric information and this is rationalised to segments of movement, which add the sources of motion information such as speed, rhythm and pattern (movement) onto physical information (static). Specifically, this work focuses on how to employ 'measurable' factors onto rules, how intentional

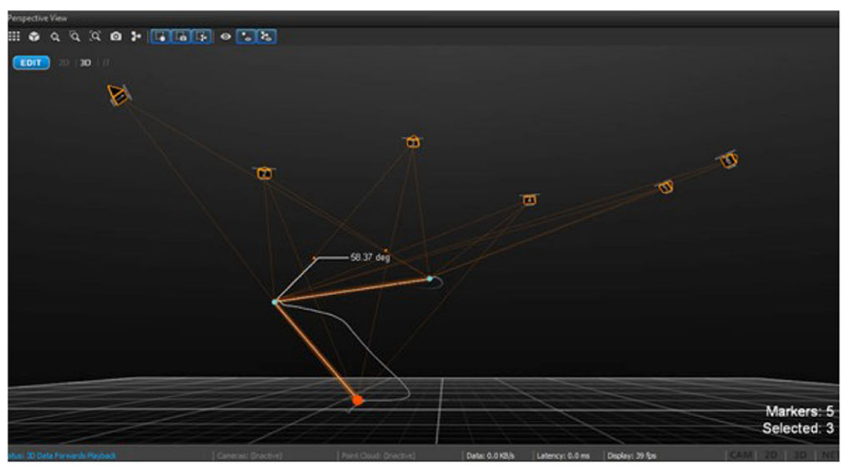

(a)

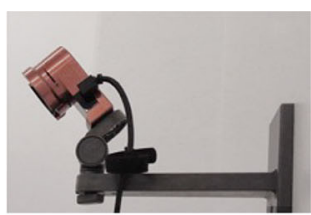

(b)

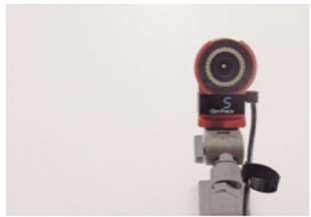

(c)

Fig. 12 a Optitrack recording display; b Optitrack camera; c Optitrack camera 
Fig. 13 Data of improvised movement

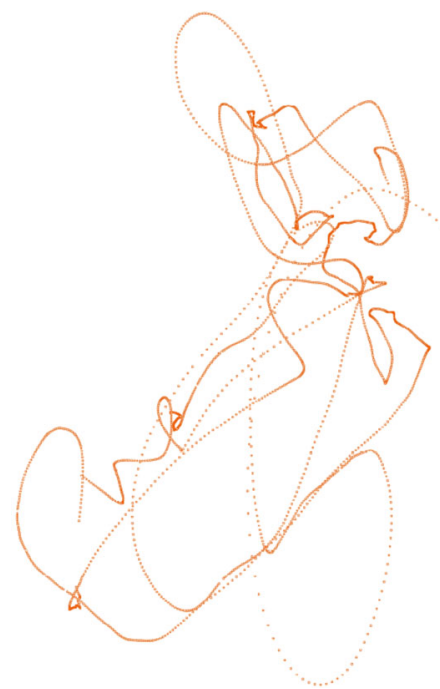

movements are transferred to the perceived 'quality' of movements through the notions of Euclidean geometric exactness and how rhythmic pattern is reviewed.

\section{Improvised Movement}

An improvised sequence is recorded, using selected parts of the dancer's body. The following image shows a hand position tracked while a dancer creates an improvised sequence. For each movement creation, the dancer is informed to use hand positions specifically in relation to the other parts of the body so that this particular part of the body leads and generates the body movement in relation to the structure that the body forms (Fig. 13).

\section{Rule-Based Movement}

Two parts of the body are recorded in the same sequence. Under this environment, the dancer is given a general rule to develop a movement using one part of the body in relation to the other (Fig. 14).

\section{Choreographed Movement}

An iterative sequence is prompted. Using a digital notation system, which is produced from the rationalisations of recorded movement, the second dancer proposes a sequence. This is recorded in the same way as the first movement is collected and further sequential development is constructed (Fig. 15).

In the process of this investigation, a dancer's 'creativity' is found notably at a point of each translation stage-from the dancer's intention to movement, sequence to digital information, recorded notation to another movement creation, and so on. Derived from the original research purpose, this 'annotative' activity seemed to 


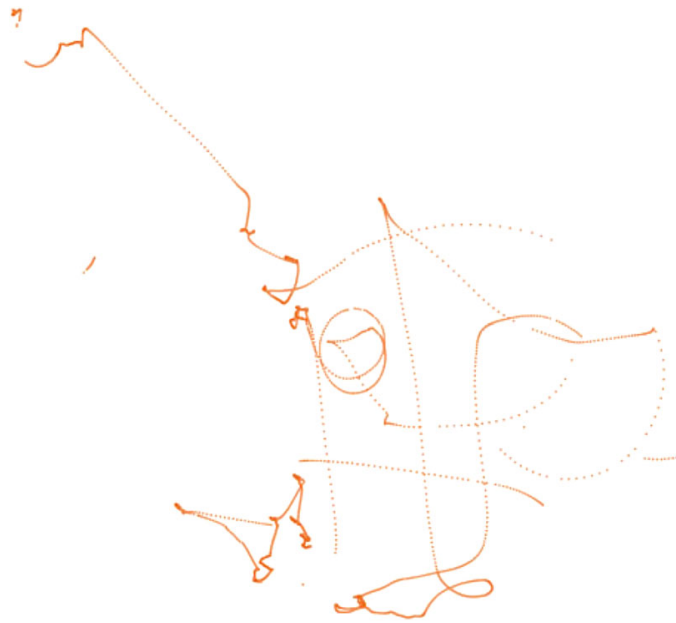

Fig. 14 Data of rule-based movement

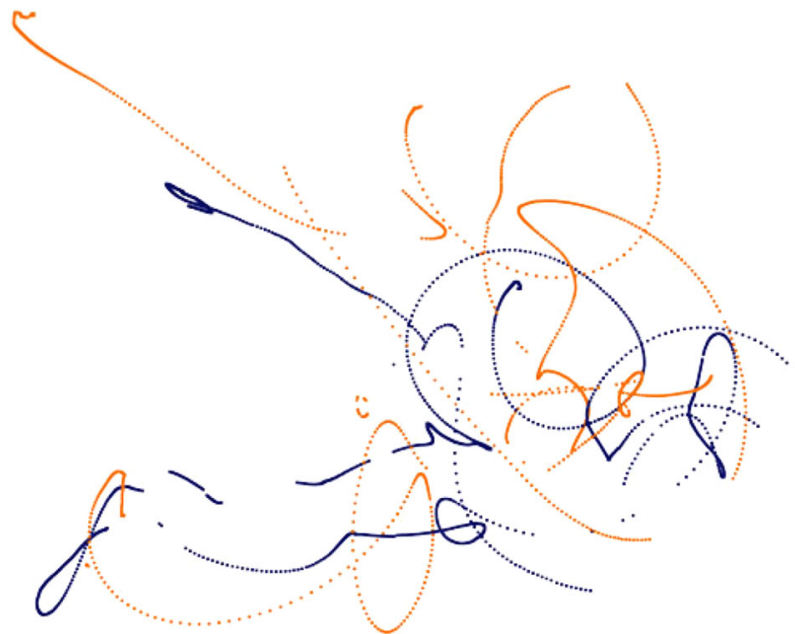

Fig. 15 Data of choreographed movement

offer dancers a creative opportunity as by-product, which is not supported in conventional 'analogue notation' systems (Fig. 16).

\section{Data-Driven Fabrication}

\section{Analogue Spatial Construction}

How can a digital notation produce architectural forms? In order to complete the circular scenario of 'performance-driven fabrication', the technical part of the 

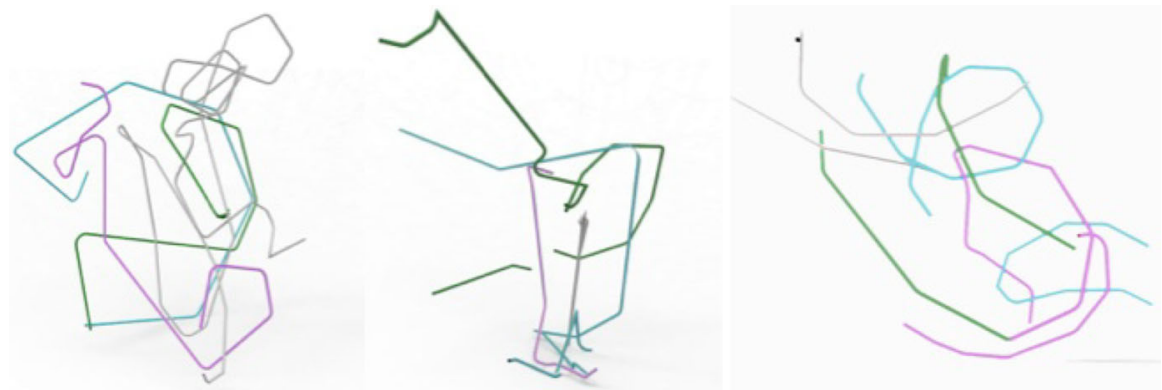

Fig. 16 Rationalised movement data (improvised/rule-based/choreographed)

physical construction phase combines readings of the digital score and automated fabrication.

Taking the physical models of 'positive space' as the outcomes of analogue dance, the aim for the fabrication phase was set to produce 'negative space' (Fig. 17). This involved another rule creation in consideration of the translation processes from digital notation to analogue space construction. The mathematical approach to transcribe body movements into 'toolpaths' for a pipe-bending tool was explored.

In comparison to the vocabularies that 'positive space' figures generate, the 'negative space' models indicated a larger interrelation between body and outer space. An applied set of rules, which were the pathway creations for materials to be

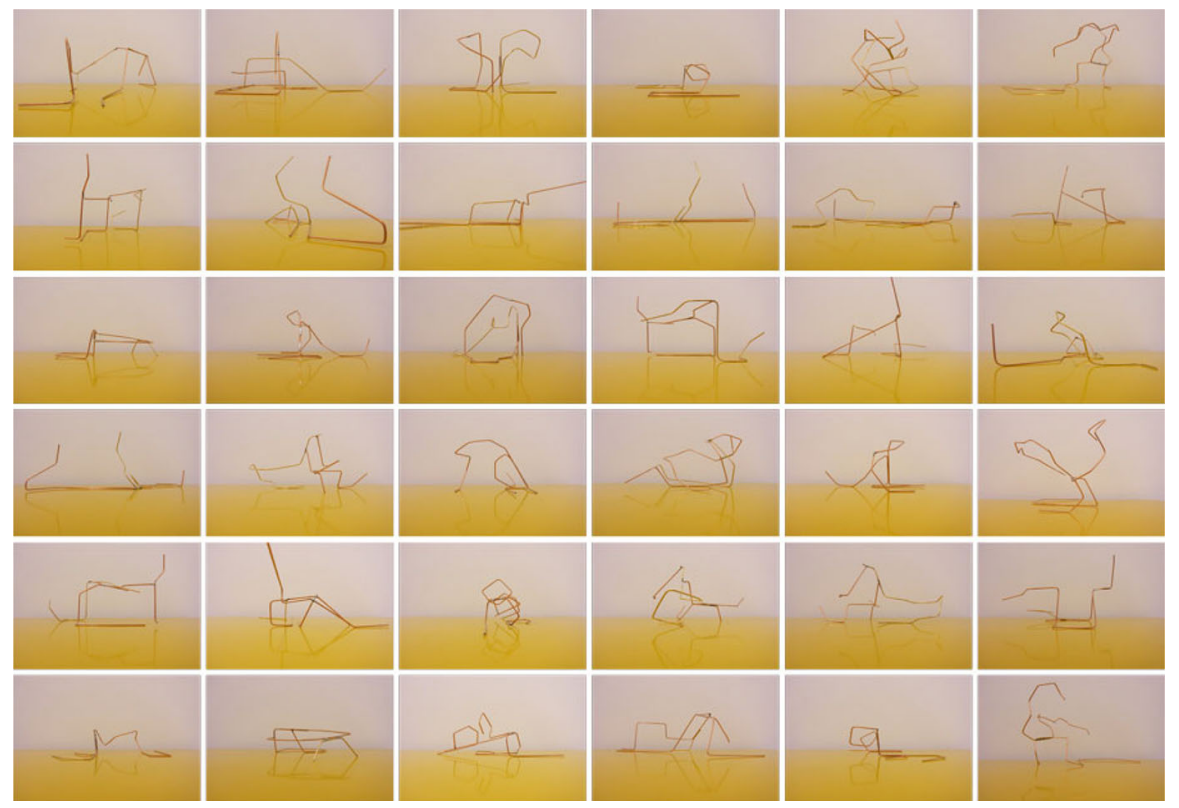

Fig. 17 Study Models form 'negative space', based on a set of rules which indicate $30^{\circ}, 45^{\circ}, 60^{\circ}, 90^{\circ}$ and rotation executed at each point of the bending process 


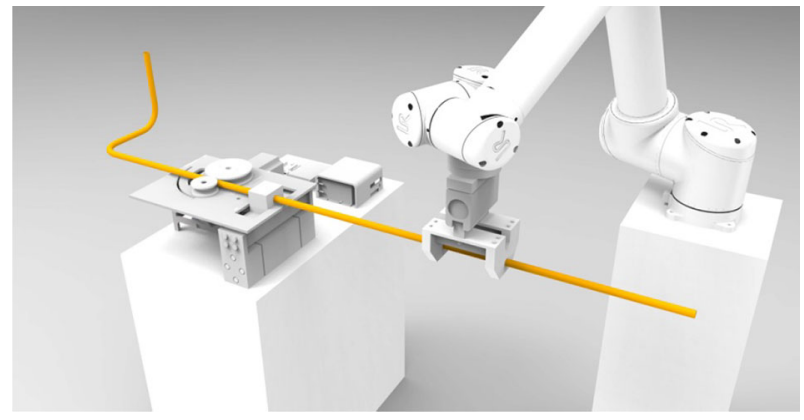

Fig. 18 Robot and bending machine (Prototype B)

bent according to a selective combination of a degree change and a rotation, assessed the logics to translate the motion to the physical forms.

The bespoke end effectors are designed for the UR10 robotic arm. The grip end effector arranges the materials within a range set in the fabrication hub, and the bending process is executed on the bending bench accordingly (Fig. 18).

Aluminium tubing was chosen as the materialisation of the notation for its ease of bending while maintaining structural capabilities. Body movements were rationalised into 'toolpaths' for robotic bending with an applied set of rules that included the constraints of material.

Rationalization of performance data enables the translation of movement into assemblies of discrete gestures (Fig. 19a, b). Bent materials are picked up from the bending machine by dancers and assembled along their performance (Fig. 20a). This process gives performers not only the memory of what has been created but also inspirations from the physical weight and volume that have been transferred from dance to object (Fig. 20b). Surrounded by the presence of outcomes and physical response to their own movements, space becomes an aggregation of moments of communication as the performance continues. Within the repetition and transfer, rhythms are created in which inhabitants can perform and occupy building areas of density and flight.

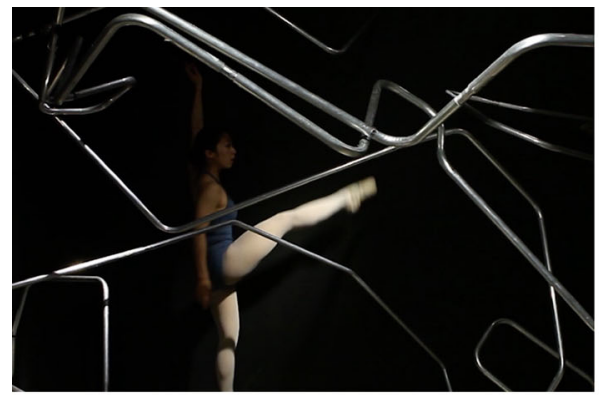

(a)

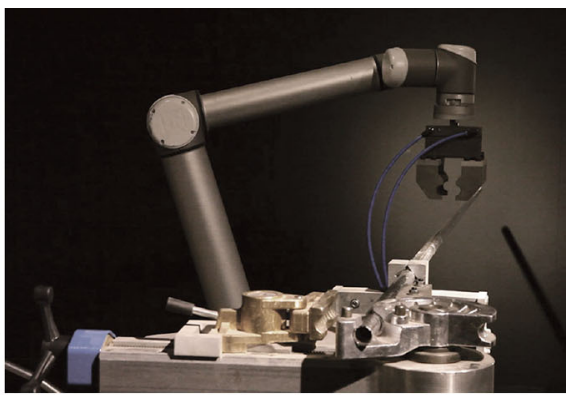

(b)

Fig. 19 a Dance movement that informs the bending system to bend aluminium pipe; b UR 10 Robot feeding the aluminium pipe to a bending machine 


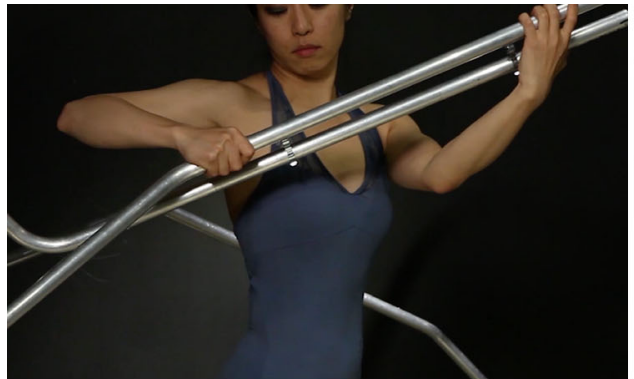

(a)

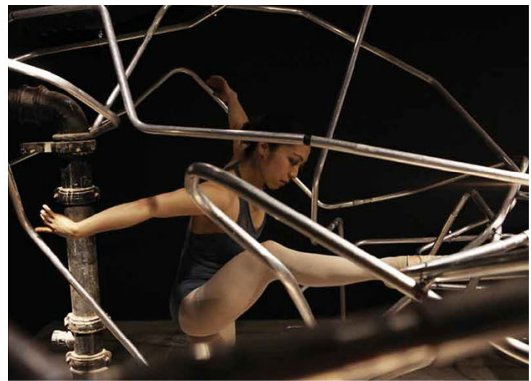

(b)

Fig. 20 a Construction of aluminium pipe by a dancer during the performance; $\mathbf{b}$ dance inspired with bent aluminium construction built along dance performance

Fig. 21 Bending system and dance performance whole view

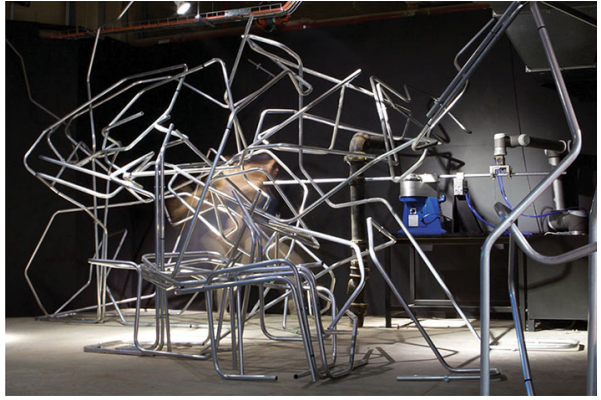

These iterative qualities of construction challenge the audience as later invited inhabitants to sense and touch the intent of a performance that emerges within the complexity of movement. During this process, the autonomy of such a system draws attention to where the expression of a performers' thinking process is focused (Groves et al. 2007).

This is the cycle of creativity, which happens in the translations and interdependency between attention, thoughts and approaches. Sharing what is happening in the procedures creates an invitation for the information to be kept and renewed. These procedures gradually grow in form, ultimately making the invisible 'quality of movement' visible. The notions of repetition, rhythm and pattern designate vital potential to qualify movement in space and raise questions of how these qualitative segments (movement) can be articulated in quantitative (physical) matter (Fig. 21).

\section{Conclusion}

This paper has presented the ways in which a digital notation system has the potential to serve as a tool of thought for architects, choreographers and artists in designing performance-driven space. It is described as an annotative condition, allowing inhabitants' participation and the creation of experience through the 
quality of changing environment. In accordance with users' behaviour and actions, these modalities are derived through their motivation. Earlier investigations by artists and architects of the close relationships between the conflicting modes of static and moving space have exposed the indispensability of this interactive modality in space.

This study has investigated the quality of movement under the distinct circumstances of two activities - in architecture, and in dance choreographydrawing upon the advantages of digital notation systems in dealing with the categories of scale, time, and the implementation of design.

Finally, the application of these concepts in a design production process describes an integrated self-processing design procedure, which generates its condition based on the inhabitants' behaviour or actions. This scenario illustrates how spatial conditions can be actively supported by events, which ultimately maintain spatial quality by holding the user's wants and needs.

Simultaneously, the example of digital data in this project, as numerically notated information, demonstrates the potential of data-driven fabrication in parallel with performance-driven design, and therefore suggests further iterative application from the example works discussed in the section "Fabrication of Movements". It supports both design and fabrication stages of the procedure to create continuous interactivity in the construction of space.

From the perspective of architects and choreographers, using movement to create an environment demonstrates how drawings and notations in both of these fields might become more dynamic communication tools. It has also demonstrated the contribution of these tools to the way artists state their creative directions. The work therefore contributes to both theory and practice, exploring connections between fundamental cognisance of body, movement and geometry relative to built and imagined spatialities.

\section{References}

Bacon, Edmund N. 1974. Design of Cities. Harmondsworth: Penguin Books.

Bonnemaison, S. and R. Eisenbach. 2009. Installations by Architects: Experiments in Building and Design. Princeton: Princeton Architectural Press.

Collard, C. 2015. Moving or Morphing Target? Hypermedial Hybrids, Diller + Scofidio-style. Body, Space, and Technology 14, Brunel University London.

Gage, S. 2007. Constructing the User. Systems Research and Behavioural Science 24(3): 313-322.

Goulthorpe, M., Burry, M., Dunlop, G. 2001. Aegis Hyposurface: The Bordering of University and Practice, Work-in-Progress, Part 1, ACADIA 2001, 344-349.

Groves, R., deLahunta, S., Zuniga-Shaw, N. 2007. Talking about Scores: William Forsythe's Vision for a New Form of 'Dance Literature'. In: Knowledge in Motion: Perspectives of Artistic and Scientific Research in Dance, TanzScripte 9, 91-100.

Kamvasinou, K. 2010. Notation Timelines and the Aesthetics of Disappearance. The Journal of Architecture, 15(4): 397-423.

Negroponte, N. 1970. The Architecture Machine: Toward a More Human Environment. Cambridge, Mass.: MIT Press.

Salingaros, N. A. 1999. Urban Space and its Information Field. Journal of Urban Design, 4(1): 29-49. Schwabe, C. 2010. Eureka and Serendipity: The Rudolf von Laban Icosahedron and Buckminster Fuller's Jitterbug. Bridges 2010, 271-278. 
Siegfried, W. 1988. Dance, the Fugitive Form of Art: Aesthetics as Behaviour. In: Rentschler, I. et al. Beauty and the Brain. Basel: Birkhäuser.

Sparacino, F. 2002. Narrative Spaces: Bridging Architecture and Entertainment via Interactive Technology. In: 6th International Conference on Generative Art, Milan, Italy.

Tschumi, B. 1996. Architecture and Disjunction, Cambridge, Mass.: MIT Press.

Weinstein, B. 2013. Performing Architectures: Closed and Open Logics of Mutable Scenes. Performing Research: A Journal of the Performing Arts 18(3): 161-168.

Syuko Kato Westby enjoys the practice of architecture as a process of realising the potential of site, including respect for its historical and environmental context, exploring possibilities and encouraging interactivity among people and the natural and built environments. She believes that integrating this changing environment in design will bring urban landscape to life. She takes inspiration from a crossdisciplinary approach, drawing on work in other fields including dance and choreography. After 11 years of professional experience working in the UK, France, and Japan, she pursued her own research interests at The Bartlett School of Architecture, from which she gained a Masters degree in 2015. Her research into interactivity and performance in architecture was introduced at the international conference "Architecture InPlay' in Portugal in 2016.

Ruairi Glynn is Director of the Interactive Architecture Lab at the Bartlett School of Architecture, UCL. Alongside his teaching and research he practices as an installation artist, recently exhibiting at the Centre Pompidou Paris, the National Art Museum of China in Beijing, and Tate Modern London. His interactive installations reflect on rapid developments in robotics, material science and computational technologies, examining the emerging aesthetics of behaviour permeating art, architecture and design. He has worked with cultural and research institutions, including the Royal Academy of Arts, the Medical Research Council and the BBC, and has built public works for Twitter, Nike, Arup, Buro Happold, and Bank of America Merrill Lynch. He has co-authored two books, 'Digital Architecture, Passages Through Hinterlands' and 'Fabricate, Making Digital Architecture,' in parallel with curating over a dozen international conferences, competitions and exhibitions. He has taught internationally, including ETH, Zurich, TU, Delft, The Royal Danish Academy of Fine Arts, Denmark and the Angewandte, Vienna. 